\title{
Trivium
}

Revue franco-allemande de sciences humaines et sociales - Deutsch-französische Zeitschrift für Geistesund Sozialwissenschaften

$30 \mid 2019$

La constitution au tournant des XXe et XXle siècles

\section{Die Konstitutionalisierung des Rechts und die Herausforderungen durch das Völkerrecht und das Europarecht}

\section{Constance Grewe et Hélène Ruiz Fabri}

Traducteur : Olivier Joop

\section{OpenEdition}

\section{Journals}

Édition électronique

URL : http://journals.openedition.org/trivium/6714

DOI : 10.4000/trivium.6714

ISSN : 1963-1820

Éditeur

Les éditions de la Maison des sciences de l'Homme

Référence électronique

Constance Grewe und Hélène Ruiz Fabri, « Die Konstitutionalisierung des Rechts und die

Herausforderungen durch das Völkerrecht und das Europarecht », Trivium [Online], 30 | 2019, online erschienen am 18 Dezember 2019, abgerufen am 10 Dezember 2020. URL : http:// journals.openedition.org/trivium/6714 ; DOI : https://doi.org/10.4000/trivium.6714

Ce document a été généré automatiquement le 10 décembre 2020.

\section{(c) (i) () $\Theta$}

Les contenus des la revue Trivium sont mis à disposition selon les termes de la Licence Creative Commons Attribution - Pas d'Utilisation Commerciale - Pas de Modification 4.0 International. 


\title{
Die Konstitutionalisierung des Rechts und die Herausforderungen durch das Völkerrecht und das Europarecht
}

\author{
Constance Grewe et Hélène Ruiz Fabri
}

Traduction : Olivier Joop

\section{NOTE DE L'ÉDITEUR}

Wir danken Frau Constance Grewe und Frau Hélène Ruiz Fabri für die freundliche Genehmigung, diesen Artikel in deutscher Übersetzung zu publizieren.

Nous remercions Mme Constance Grewe et Mme Hélène Ruiz Fabri de nous avoir accordé l'autorisation de traduire ce texte pour le présent numéro.

1 Konstitutionalisierung, Europäisierung, Internationalisierung ... So viele bestehende oder sich abzeichnende Tendenzen, die durch ihre Neuheit bestechen und durch ihre Dynamik Interesse erwecken. Doch, wie Jean-Claude Gautron gerne seinen Doktoranden zu verstehen gibt, »nicht alles, was glänzt, ist Gold«, eine Einstellung, die sich auch in seiner eigenen Haltung der »kritischen Betrachtung Europas durch einen Europarechtler ${ }^{1}{ }^{1}$ widerspiegelt. Ihn durch den Versuch zu würdigen, die Verwendung bestimmter Begriffe zu beleuchten, ist daher nicht unangemessen.

2 1. Der bereits von Boris Mirkin-Getzewitsch ${ }^{2}$ geprägte und insbesondere von Louis Favoreu und Joël Rideau 3 übernommene Begriff des »droit constitutionnel international«, des international ausgerichteten Teils des Verfassungsrechts, bezeichnet die Bestimmungen der Verfassung, die sich auf die auswärtigen Beziehungen und die Öffnung des nationalen Rechts zum Völkerrecht beziehen. Im Zuge der deutlichen Zunahme derartiger Bestimmungen in den Verfassungen der Staaten Europas, insbesondere als Folge des Vertrags von Maastricht, und der Angleichung des 
materiellen Rechts infolge des Einflusses der Rechtsprechung des EuGH und des EGMR auf das innerstaatliche Recht und die innerstaatliche Rechtsprechung, ist in der Rechtssprache der Begriff des droit constitutionnel international fortschreitend verdrängt worden durch den Ausdruck der Internationalisierung beziehungsweise Europäisierung des Verfassungsrechts ${ }^{4}$ - ein Ausdruck, der, je nach Betrachtungswinkel, die Vorderoder Rückseite ein- und derselben Medaille darstellt, und dessen semantisches Gegenstück die Konstitutionalisierung des Völker- und/oder des Europarechts darstellt.

3 An dieser Stelle könnte leicht der Eindruck entstehen, dass dieses Begriffspaar Internationalisierung bzw. Europäisierung auf der einen und Konstitutionalisierung auf der anderen Seite - auf zwei unterschiedliche Aspekte der gegenwärtigen Entwicklungen des Rechts verweisen würde, die im Wettbewerb oder gar im Konflikt miteinander stehen. Jedoch steht bei einer stärker auf die Wechselwirkung und die Vielfalt der betrachteten Rechtsordnungen, im Besonderen in Europa, ausgerichteten Analyse der »Netzwerk-« oder »multi-level government«-Charakter ${ }^{5}$ des Verbundes von innerstaatlichem und europäischem Recht im Vordergrund. Bei einer solchen Betrachtungsweise befinden sich beide Rechtsordnungen in einem Prozess der gegenseitigen Annäherung, Vernetzung und Verflechtung, sodass die Völker- und Europarechtsfreundlichkeit des Verfassungsrechts als Teil dieser Konstitutionalisierung zutage tritt. ${ }^{6}$ Die Internationalisierung/Europäisierung des Verfassungsrechts stünde damit der Konstitutionalisierung des Völker-/Europarechts nicht entgegen, sondern stellte im Gegenteil ein Element desselben dar. Allerdings muss man sich darüber klar sein, was man mit diesen Begriffen wirklich bezeichnet.

4 2. In einem ersten Ansatz kann davon ausgegangen werden, dass der Begriff der Konstitutionalisierung den Vorgang beschreibt, durch den eine Vorschrift Teil der Verfassung wird oder Verfassungsrang erlangt. Zunächst in der deutschsprachigen und der anglo-amerikanischen ${ }^{7}$ Rechtsliteratur in Bezug auf das Völkerrecht und das Europarecht gebraucht, ist der Terminus von der französischen Rechtslehre aufgegriffen worden. Diese hatte den Begriff allerdings schon auf innerstaatlicher Ebene verwendet, um die seit 1971 eingetretene neue Qualität des Vorrangs und des normativen Charakters der Vorschriften der Verfassung zu bezeichnen. So ist bereits von einer Konstitutionalisierung des Strafrechts, des Zivilrechts oder des Verwaltungsrechts im Sinne eines Hineinwirkens des Verfassungsrechts in diese Rechtsbereiche gesprochen worden. ${ }^{8}$

5 Ist allerdings dieselbe Art von Konstitutionalisierung gemeint, wenn von einer Konstitutionalisierung des Völkerrechts oder des Europarechts die Rede ist? Zweifel sind angebracht.

6 Zum einen ist der französische Begriff "constitution « nicht deckungsgleich mit dem englischen »constitution« oder dem deutschen »Verfassung«. Im Englischen und im Deutschen hat der Begriff viel mehr als im Französischen seine ursprüngliche etymologische Bedeutung beibehalten, im Sinne der Schaffung grundlegender Statuten eines Organs oder einer Institution. Jean-Claude Gautron hat von der ironischen Bemerkung Peter Hains, des Beraters von Tony Blair, berichtet, der angemerkt habe, auch sein Golfverein besitze eine Verfassung. ${ }^{9}$ Im deutschen Recht taucht die Bezeichnung "Verfassung" auch mit Bezug auf Unternehmen oder Gemeinden auf, wohingegen sie im Französischen im Wesentlichen auf den Prozess beschränkt bleibt, in dessen Rahmen ein Staat oder eine internationale Organisation geschaffen wird. Vor 
diesem Hintergrund ist es möglich oder gar wahrscheinlich, dass, je nach nationaler Rechtslehre, eher mehr oder eher weniger Hemmungen bestehen, diesen Begriff und dessen Ableitungen $\mathrm{zu}$ verwenden. Dies schließt freilich nicht aus, dass die Verwendung dieses Begriffes mit unterschiedlichen rechtlichen Traditionen oder zumindest mit einer Rechtsauffassung, die ein anderes Verständnis des Verfassungsbegriffes aufweist, verknüpft ist.

7 Zum anderen liegt der Vorteil des Begriffes "Konstitutionalisierung«, wie bei allen Bezeichnungen mit der Endung "-isierung ${ }^{10}$, in seiner Mehrdeutigkeit, die sowohl einen Zustand als auch den Prozess zu dessen Entstehen beschreiben kann. Er kann auch dann verwendet werden, wenn dieser Prozess noch lange nicht abgeschlossen ist, und er kann sogar präskriptiv gebraucht werden. ${ }^{11}$ Gleichwohl ist eine prospektive Verwendung des Begriffes natürlich mehrdeutiger als eine rückblickende, birgt sie doch die Gefahr, als Versuch gewertet zu werden, gegenwärtige Entwicklungen am Maßstab eines naturgemäß politischen Vorhabens deuten zu wollen.

Schließlich vermischt eine solche Analyse unvermeidlich das Recht und den Diskurs über das Recht. Diese Feststellung gilt noch mehr im Hinblick auf das Europarecht als auf das Völkerrecht im Allgemeinen, da der Konstitutionalisierungsbegriff im Europarecht über den Diskurs in der Rechtslehre schon lange hinausgewachsen ist und es für die Literatur nicht mehr so sehr darum geht, den Dingen einen Namen zu geben, als Stellung zu beziehen zu den Begrifflichkeiten, die diesen Dingen von der Politik gegeben wurden, und anschließend die Stichhaltigkeit des zugrundeliegenden Gedankengangs $\mathrm{zu}$ bewerten. Diesbezüglich führt die Verschränkung der formal-, materiell- und verfahrensrechtlichen Aspekte des Begriffes der Verfassung zweifelsohne zu Vielschichtigkeit und Verwirrung.

Dies ist der Hintergrund für die nachfolgenden Überlegungen über die Bedeutung der Übernahme des Begriffs der Konstitutionalisierung im französischen Rechtsdiskurs zur Beschreibung eines dem Völkerrecht und dem Europarecht eigenen Phänomens. Hier stellt sich die Frage, ob es sich bei dieser terminologischen Übernahme um ein fehlgeleitetes oder im Gegenteil fruchtbares "Nachplappern" oder um einen surrealistischen Schritt handelt, der - angelehnt an Magrittes Behauptung, bei der von ihm gezeichneten Pfeife handele es sich nicht um eine Pfeife - in umgekehrter Vorgehensweise behauptet, "dies ist eine Verfassung " und somit Teil eines politischen Projektes ist. Eine einzige und eindeutige Antwort auf diese Fragen erscheint schwierig, aber es stellt sich dennoch heraus, dass das Phänomen der "Konstitutionalisierung" nicht völlig neu ist; es ist die Eindringlichkeit dieser Erscheinung, die eine neue Stufe erreicht hat und somit die Veränderung der Bezeichnung erklärt bzw. dessen, was die Sprachwissenschaft mit dem Begriff des Signifikanten oder Bezeichnenden umschreibt (I). Spiegelt diese Veränderung des Signifikanten lediglich eine quantitative Entwicklung wider oder ist sie Ausdruck eines tatsächlichen qualitativen Sprunges; anders ausgedrückt, schließt die Veränderung des Bezeichnenden auch eine Veränderung des Bezeichneten, des Signifikats, mit ein (II)?

\section{Der Wandel des Bezeichnenden}

Heutzutage ist die Konstitutionalisierung oder zumindest die Debatte über diese Konstitutionalisierung im Europarecht weiter vorangeschritten als im Bereich des Völkerrechts, doch ist es Letzteres, in dem die zu behandelnden Fragen, Überlegungen 
und Prognosen zuerst Bedeutung erlangten. Dies liegt nicht ausschließlich an der logischen Feststellung, dass das Völkerrecht bereits vor dem Europarecht bestand (noch ist dieses nicht von jenem getrennt). Tatsächlich können zwei Phasen der Konstitutionalisierungsdebatte unterschieden werden. Die erste setzte nach dem Ersten Weltkrieg ein und speiste sich aus dem Reichtum der damals entstehenden Normengefüge und Strömungen in der Rechtslehre. Die zweite ist deutlich jünger und behandelt erkennbar das Europarecht vor dem »übrigen« Völkerrecht (wobei die Frage nicht erörtert wird, ob das Europarecht noch immer als Teil des Völkerrechts oder als eigenständige Rechtsordnung angesehen werden kann/soll).

\section{A. Eine im Völkerrecht bereits seit Längerem bestehende Erscheinung}

11 Dieses Alter ist freilich relativ: Die Wurzeln liegen im Wesentlichen zu Beginn des 20. Jahrhunderts. Das Phänomen der Konstitutionalisierung findet seinen Niederschlag hauptsächlich in der Rechtswissenschaft, und in mehrdeutigerer Weise im Recht selbst. Der historische Hintergrund ist alles andere als unbedeutend, insbesondere im Hinblick auf zwei Aspekte. Zum einen entstand nach dem Ersten Weltkrieg in der Rechtslehre eine Gegenbewegung zur positivistischen Strömung, die ihren Niederschlag auch in der Vorstellung vom Verfassungsbegriff finden sollte. Zum anderen führte die Notwendigkeit einer Zusammenarbeit in bestimmten Bereichen, insbesondere der Kommunikation, zu verstärkten kollektiven Ansätzen und im Besonderen zu neuen internationalen Einrichtungen, was eine neue Ausgestaltung von Teilordnungen der internationalen Beziehungen und einen Reflexionsprozess über Sinn und Folgen dieser Neuausgestaltung auslöste.

\section{Der Traum der Rechtslehre von einer Verfassung der Völker}

12 Die Formulierung mag etwas radikal erscheinen, so wie es wohl jede Zusammenfassung verschiedener Rechtsauffassungen in einer kurzen Formel sein muss. Auch auf die Gefahr einer übertrieben vereinfachenden Darstellungsweise hin kann behauptet werden, dass eine der Gemeinsamkeiten der Lehrmeinungen nach dem Ersten Weltkrieg darin bestand, das Völkerrecht gewissermaßen als »Welt-Recht ${ }^{12}{ }^{12} \mathrm{zu}$ denken. Sofern in diesem Kontext der Begriff der Verfassung auftauchte, so geschah dies weit entfernt von und in ganz unterschiedlicher Weise als bei demjenigen Ansatz, der das Völkerrecht auf den die Außenbeziehungen der Staaten regelnden Teil des öffentlichen Rechts verengte und dieses die auswärtigen Beziehungen regelnde öffentliche Recht lediglich als Teilbereich des jeweiligen nationalen Verfassungsrechts ansah. ${ }^{13} \mathrm{Im}$ Gegenteil schimmerte hier im Hintergrund die Idee einer "Gesellschaft der Völker" durch, bei der es nicht lediglich um ein einfaches Nebeneinander der Staaten und ein "allgemeines « Völkerrecht zur Friedenswahrung ging. ${ }^{14}$ Die Herausbildung der Idee einer Verfassung steht im Zusammenhang mit der Suche nach neuen Denkmustern, um eine Grundlage des Rechts außerhalb ausschließlicher Willensentscheidungen, also gewissermaßen »in etwas Objektivem « zu finden. "Eine Verfassung zu postulieren bedeutete, das Völkerrecht auf einer objektiven Grundlage zu verankern, die, ebenso wie eine Verfassung auf nationaler Ebene, geeigneter war, Macht und Recht miteinander in Einklang zu bringen." Der Begriff der Verfassung wurde also als Bezeichnung für »die Organisation der Gewalten und der grundlegenden 
Zuständigkeiten« verstanden. ${ }^{15}$ Dieser allgemeine Ansatz untergliederte sich jedoch in verschiedene Strömungen.

Die Anhänger eines soziologischen Ansatzes in der Rechtslehre vertraten den Gedanken, dass jede Organisation oder Institution über eine Verfassung verfüge. $\mathrm{Zu}$ diesen Anhängern zählten einige Vertreter der italienischen Schule, ${ }^{16}$ vor allem jedoch Verfechter des sogenannten Solidarismus, wie Georges Scelle oder Léon Duguit. Sie glaubten nicht an eine Zukunft für die Staaten, sondern vertraten die Auffassung, die geschichtliche Entwicklung gehe in Richtung der Entstehung einer Weltgesellschaft, in deren Rahmen die Einzelnen die Rechtssubjekte des Völkerrechtes würden. ${ }^{17}$ Nationales Recht und Völkerrecht würden gewissermaßen zum Wohle des gesellschaftlichen Fortschritts zu einer Einheit verschmelzen. Die Leitidee dieser Autoren war daher die Verfassung, die sie auf die soziale Realität oder die politische Gesellschaft bezogen. Dabei wurde auch das Problem der Verbindung von Verfassung und Staatswesen, allerdings mittels eines sehr ungenauen theoretischen Konstrukts, aufgelöst. Aus formeller Sicht zeichnete sich deren Herangehensweise durch die Verwendung von aus dem Verfassungsrecht entlehnten Begriffen und Vorstellungen aus, um das Völkerrecht zu untersuchen (so verwendete G. Scelle z. B. die Begriffe Föderalismus, Gesetzgebungsfunktion, exekutive Funktion, Rechtsprechungstätigkeit) und gegebenenfalls, nach Maßgabe der Anforderungen, die diesen Begriffen zugeordnet werden, noch Mängel des Völkerrechts hervorzuheben. Allerdings geschah dies zu einer Zeit, in der der Begriff der Verfassung selbst noch nicht so »bestimmt « war wie heute, und in der viele nationale Verfassungen sich gerade anschickten, sich ihren normativen Charakter zu erkämpfen. In der deutschsprachigen Rechtslehre vertrat in jener Zeit Alfred Verdross ähnliche Auffassungen und befürwortete die Idee einer Verfassung für die Völkerrechtsgesellschaft, ${ }^{18}$ deren Vorschriften zugleich Ausgangspunkt und Legitimationsgrundlage - mit einem Wort, die Grundnorm - des Völkerrechts bilden sollten. Gleichzeitig gesellten sich zu diesen Gedanken über Kompetenzverteilungen auch Überlegungen $\mathrm{zu}$ materiell-rechtlichen Fragen wie derjenigen der Gerechtigkeit oder des Gemeinwohls hinzu, was dazu führt, dass Verdross' Ideen eher einer Wiederbelebung des Naturrechtsgedankens zugeordnet werden können.

14 In gewisser Hinsicht stellt sich die Frage, ob die Verwendung des Verfassungsbegriffs nicht Ausdruck einer Ausuferung oder einer Wandlung der Debatte über die Eigenschaft des Völkerrechts als Rechtsordnung ist, einer Debatte, die noch lange nicht erschöpft ist ${ }^{19}$ und sogar eine gewisse Wiederbelebung erfährt, obwohl es schien, dass sich die neuere Rechtslehre zum Teil von ihr abgewendet hätte. ${ }^{20}$ Jedenfalls hat man das Gefühl, nie weit davon entfernt zu sein. In diesem Zusammenhang kann es interessant sein festzustellen, dass diese Entwicklung zusammenfällt mit einer vertieften Beschäftigung mit der Frage des Verhältnisses von Völkerrecht und innerstaatlichem Recht. Vielleicht nicht das Völkerrecht selbst, aber zumindest die Völkerrechtslehre setzt sich mit dem innerstaatlichen Verfassungsrecht auseinander, ebenso wie die Staatsrechtslehre auch das Völkerrecht behandelt. Bezeichnenderweise sehen diejenigen Denkströmungen, die am ehesten geneigt sind, den Verfassungsbegriff auch in Bezug auf das Völkerrecht zu verwenden, dieses Verhältnis als hierarchische Beziehung zwischen übergeordnetem Völkerrecht und untergeordnetem Staatsrecht an (bzw. fassen diesen Zustand als Übergangszustand bis zur Entstehung eines rein monistischen Systems auf). 

einem Institutionengefüge dieser internationalen Gesellschaft, die sich als solche wahrnimmt, begünstigt oder genährt.

\section{Die Gründungsverträge internationaler Organisationen aus verfassungsrechtlicher Perspektive}

16 wenn sie historisch betrachtet mit der Entwicklung internationaler Organisationen zusammenfällt. Sie gründet auf einem Verständnis des Verfassungsbegriffes wie es im Englischen und im Deutschen möglich ist (s. oben in der Einführung) und ist vor allem darauf gerichtet, die internen Strukturen der Organisation zu untersuchen. Hier kann auch von einer "mikroverfassungsrechtlichen ${ }^{21}$ Analyse gesprochen werden. Der Gründungsvertrag einer internationalen Organisation markiert die Schaffung eines neuen Gebildes, bestimmt dessen Organe, legt die Verteilung der Zuständigkeiten fest, ermächtigt zu bestimmten Handlungen und begründet die Gültigkeit der Rechtsakte, die im weiteren Verlauf innerhalb und von der Organisation angenommen werden. Dieser Gründungsakt kann sogar ausdrücklich den Namen Verfassung tragen, dies ist beispielsweise bei der Internationalen Arbeitsorganisation (ILO) der Fall. Dieser Betrachtungsweise kann auch die Entwicklung der Analyse des Europarechts, als Recht einer besonderen internationalen Organisation, in verfassungsrechtlichen Kategorien zugeordnet werden. In diesem Sinne stellt eine solche Analyse nicht zwangsläufig ein Argument zugunsten einer Abspaltung und Sonderstellung des Europarechts gegenüber dem Völkerrecht dar, zumal besagte Analyse einige Wesensmerkmale der Gründungsverträge internationaler Organisationen selbst mit einbezieht - und somit als auch für das Europarecht geltend anerkennt: das Merkmal, dass die Gründungsverträge häufig einen Vorrang gegenüber anderen oder sogar allen anderen Verträgen beanspruchen, die die Mitgliedstaaten oder auch die Organisation selbst abschließen, das Merkmal, dass eine Annahme der Gründungsverträge nur mit allen deren Bestandteilen, d. h. ohne Vorbehalte, zulässig ist, sowie das Merkmal, dass mit den Gründungsverträgen spezifische Herausforderungen bei deren Auslegung einhergehen..$^{22}$ Diese Betrachtungsweise des »internationalen Verfassungsrechts « ist oftmals allerdings mit weitergehenden Bestrebungen verbunden, die die zweite Ebene der Analyse der Gründungsverträge internationaler Organisationen unter verfassungsrechtlicher Perspektive bilden.

Diese zweite Ebene richtet den Fokus auf die äußere Ordnung, in die sich die Organisation einbettet, und birgt eher Züge einer "makroverfassungsrechtlichen « ${ }^{23}$ Analyse. Sie betrachtet die untersuchte internationale Organisation gewissermaßen als Entwurf für das Institutionengefüge der Gesellschaft der Völker in deren Gesamtheit, insbesondere wenn die betreffende internationale Organisation ein System der kollektiven Sicherheit einrichtet und einen weltweiten Geltungsanspruch erhebt. Eine solche Analyse versucht Lauterpacht in Bezug auf die Satzung des Völkerbundes. ${ }^{24}$ Ausgehend davon, dass die Satzung analog zu innerstaatlichem Recht betrachtet werden könne, sieht er in ihr »das Grundgesetz der Gesellschaft der Völker« und vertritt die Auffassung, der verfassungsrechtliche Charakter der Satzung sei in deren Artikel 20 selbst ausdrücklich bekräftigt, der, nach Lauterpacht, »den absoluten Vorrang der Satzung gegenüber jedem anderen unter den Mitgliedern des Bundes geschlossenen Abkommen ${ }^{25}$ festschreibt. Daher könne der Völkerbund nicht als eine 
einfache Organisation zur Abstimmung der diplomatischen Handlungen ihrer Mitglieder angesehen werden, insbesondere da seine Satzung »ein System der kollektiven Sicherheit [einrichtet], das bei näherer Betrachtung nichts anderes [ist] als der Ausdruck der tatsächlichen Herrschaft des Rechts zwischen den Staaten, ebenso wie im Gegenteil das Fehlen eines kollektiven Sicherheitssystems das Maß für die Defizite des Völkerrechts als Rechtsordnung ${ }^{26}$ aufzeige. Konkret angewendet, schließt sich diese Vorgehensweise derjenigen an, welche die Eigenschaft des Völkerrechts als System, als normative Ordnung belegen will. Lauterpachts Analyse kann der Konfrontation mit einer Realität, die schwerlich mit den Forderungen der Völkerbundsatzung übereinstimmt, allerdings nur dann standhalten, wenn die Praxis nicht als Verstoß gegen die Satzung, sondern als Ausdruck des den Staaten von der Satzung selbst für deren Auslegung zugebilligten Ermessensspielraums verstanden wird, womit der betreffenden "Verfassung « eine eher richtungsweisende Funktion zugeschrieben wird. Gleichwohl nahm Lauterpachts Analyse diejenigen vorweg, die später in Bezug auf die Charta der Vereinten Nationen unternommen werden sollten.

\section{B. Die Herausbildung einer neuen Terminologie}

In der zweiten Hälfte des 20.Jahrhunderts entwickelte sich die Frage der Konstitutionalisierung des Völker- und des Europarechts in verschiedene Richtungen, ohne die französische Rechtslehre dabei jedoch wirklich zu erreichen.

\section{Im Völkerrecht}

Im Völkerrecht ging diese Entwicklung nicht auf systematische, sondern auf eher ungeordneten Wegen vonstatten. Dies wird besonders in zwei Bereichen und einem Graubereich deutlich.

Der erste greift die Leitlinien der Analysen aus den 1920er und 1930er Jahren auf. Spuren davon finden sich besonders in der deutschsprachigen Rechtsliteratur. ${ }^{27}$ Hier wurden Gedanken, die Verdross selbst in Bezug auf die Charta der Vereinten Nationen aktualisiert hatte, ${ }^{28}$ aufgegriffen und weiterentwickelt. Mit unterschiedlichen Akzentsetzungen wird darin nach der verfassungsartigen Funktion der Charta »im Hinblick auf die internationale Rechtsordnung in ihrer Gesamtheit ${ }^{29}$ gefragt. Diese „Verfassung", die nach dieser Einschätzung von der Charta - von der es heißt, sie habe das allgemeine Völkerrecht integriert - verkörpert wird, oder deren Teil sie ist, wird als Grundgesetz der internationalen Gemeinschaft gesehen. Nach dieser Lesart sind die Organe der Vereinten Nationen dazu berufen, diese Weltgemeinschaft zu verkörpern, und das in der Charta enthaltene Primärrecht soll gegenüber jeder anderen Verpflichtung Vorrang haben, was im Übrigen Artikel 103 der Charta explizit vorsieht. Darüber hinaus wird mit dem Begriff der »Konstitutionalisierung" des Völkerrechts auch die Tendenz zur Institutionalisierung bezeichnet, sowie die zunehmende Entwicklung multilateraler Verträge mit Weltgeltungsanspruch, die allgemeine und abstrakte Normen beinhalten, die für alle Staaten gleichermaßen gelten sollen und das internationale Völkervertragsrecht hin zu einer Art "Gesetz « ${ }^{30}$ entwickeln. Diese Form von Konstitutionalisierung - dieser global constitutionalism ${ }^{31}$ - hält den Wert der Gleichheit hoch, und die Fragen, die sie aufwirft, betreffen ihr Verhältnis zur und ihre Auswirkungen auf die Souveränität der Staaten. ${ }^{32}$ 
21 Den Graubereich bildet ein begrifflicher und normativer Nebel, der das Völkerrecht in die Richtung verfassungsrechtlicher Problemstellungen treibt. Diese Entwicklung weist Parallelen mit derjenigen der innerstaatlichen Verfassungen auf. Diese innerstaatliche verfassungsrechtliche Entwicklung zeichnet sich, insbesondere nach und seit dem Zweiten Weltkrieg, durch eine langsame und stetige Festigung der materiellrechtlichen Ebene des Rechtsstaats aus. Es geht nicht nur darum, dass der Staat dem Recht unterworfen ist, sondern dieses Recht muss eine bestimmte Qualität besitzen und Träger bestimmter Werte sein, in denen im Besonderen die Grundrechte des Einzelnen verankert sind. ${ }^{33}$ Auf der internationalen Ebene wird diese Entwicklung in materiellrechtlicher Hinsicht gespiegelt durch Menschenrechtserklärungen. In verfahrensrechtlicher Hinsicht zudem durch die Tatsache, dass die diese Menschenrechte absichernden Verträge bestimmte Wesenszüge aufweisen (insbesondere z. B. das Fehlen von Reziprozitätsklauseln), die ihnen einen stärkeren objektiv-rechtlichen Charakter verleihen. In struktureller Hinsicht zeigt sich das an der Förderung von Vorstellungen - selbst wenn deren Realisierung in der Praxis unvollendet bleibt - wie derjenigen der internationalen Normenhierarchie oder eines völkerrechtlichen ordre public ${ }^{34}$, sowie von Ideen wie derjenigen zwingender Rechtsnormen (ius cogens) oder erga omnes geltender Vorschriften, oder auch von gemeinsamen Definitionen zu ahndender Verbrechen sowie schließlich durch die Förderung des Gedankens einer internationalen Gemeinschaft selbst. Der Begriff des Rechtsstaates selbst tritt erst später, ungefähr ab den 1980er und 1990er Jahren, in Erscheinung, und dann auch zunächst weniger auf das Völkerrecht selbst ausgerichtet, sondern mehr als eine Handlungsanweisung des Völkerrechts an die nationalen Rechtsordnungen. ${ }^{35}$ Die Umwälzungen in Osteuropa und die damit verbundene Geburt oder, mit Hilfe der Staatengemeinschaft, Wiedergeburt zahlreicher Staaten haben ein bestimmtes Staatsmodell begünstigt, nämlich dasjenige eines Staates, der demokratisch verfasst ist und die Menschenrechte achtet, ${ }^{36}$ Voraussetzungen, die auch aus einer wirtschaftswissenschaftlichen Perspektive nicht infrage gestellt, sondern im Gegenteil als eine der Grundlagen einer "guten Regierungsführung" angesehen werden. Gleichwohl können diese Entwicklungen nicht als Kronzeugen für eine auf einen Konstitutionalisierungsprozess gerichtete Analyse herangezogen werden, da der Verfassungsbegriff im Rahmen des rechtswissenschaftlichen Diskurses über diese Entwicklungen praktisch nicht vorkommt. Dies kann auf eine Schwierigkeit hindeuten, die Verwendung des Begriffs vom Vorhandensein eines Institutionengefüges zu lösen, was wahrscheinlich vor allem eine Folge einer realistischen Betrachtungsweise sein dürfte, die den Fokus auf das ausschlaggebende Wesen von Machtverhältnissen legt. Es scheint unbestritten, dass die Gegnerschaft der Großmächte ursächlich dafür war, dass die Charta der Vereinten Nationen nicht die "tatsächliche Verfassung der Menschheit« ${ }^{37}$ geworden ist, zu der sie hätte werden können. Der Gedanke, dass sich die Charta diesem Ideal annähert, lebte erst im Zuge der dafür günstigen Verhältnisse nach dem Ende des Kalten Krieges wieder auf, als eine neue Weltordnung entstand.

Doch die Debatte hat, parallel und fast heimlich, noch eine weitere Facette hervorgebracht, die sich im Laufe der 1990er Jahre durchgesetzt hat, nämlich das Nachdenken über eine Globalisierung des Rechts. Der Begriff der Konstitutionalisierung nimmt darin keinen hervorgehobenen Platz ein und stellt auch kein den Diskurs strukturierendes Element dar. Er schimmert nichtsdestotrotz auf zwei sich ergänzende Arten durch, deren Gemeinsamkeit möglicherweise in der Bildung eines Gegenpols zur »Fragmentierung« des Völkerrechts besteht, zumindest jedoch in der Betonung der 
wirtschaftlichen Komponente. $\mathrm{Zu}$ diesem Bereich gehören Überlegungen, die, nach einer Betrachtung der Entwicklungstendenzen der Globalisierung des Rechts, eine Konstitutionalisierung dieses Rechts fordern, um dadurch Ordnung und Einheitlichkeit zu sichern. Eine solche Analyse verfährt ähnlich dem Prinzip des Syllogismus: „Die wesentlichen Merkmale eines Rechtsstaates und einer rechtmäßigen Ausübung von Machtbefugnissen sollten auch im Rahmen der Globalisierung gelten«. Gleichzeitig gilt: »Die Globalisierung des Rechts führt zum Teil dazu, dass diese Grundsätze nicht mehr auf der bloßen staatlichen Ebene gewährleistet werden können." Daraus ergibt sich der Schluss, dass, wenn diese »Verfassungsgrundsätze« den staatlichen Rahmen sprengen, »es notwendig wird, das Völkerrecht zu konstitutionalisieren « ${ }^{38}$

Diesem Bereich können allerdings auch Untersuchungen zugeordnet werden, die sich spezifischer mit der Welthandelsorganisation befassen und eine Konstitutionalisierung des WTO-Rechts befürworten. Diesem Recht sollte verfassungsmäßiger Charakter zukommen, ${ }^{39}$ sei es, um dadurch den Vorrang zu begründen, den es haben sollte, und/ oder sei es, um die Beachtung zu rechtfertigen, die es dem, was man unter dem Oberbegriff nichtkommerzielle Anliegen zusammenfassen könnte, schenkt oder schenken sollte, und/oder sei es schließlich, um die wirtschaftlichen Freiheiten, die es gewährleistet, in den Rang von grundlegenden Freiheiten zu erheben. Während wirtschaftliche Verhaltensweisen nicht mehr isoliert von den weiteren Erwägungen betrachtet werden könnten, die Handlungen in diesem Bereich begründen und beeinflussen, habe die WTO, namentlich wegen ihres Streitschlichtungsverfahrens, ${ }^{40}$ das Potenzial, um zur Speerspitze einer "Konstitutionalisierung « des Völkerrechts ${ }^{41} \mathrm{zu}$ werden. Der von der Rechtslehre verfolgte - im vorliegenden Fall liberale bzw. ultraliberale - Ansatz weist in seinem Aufbau gewisse Entsprechungen zur Entwicklung der Europäischen Gemeinschaft auf, die zunächst auf den Grundsätzen des freien Verkehrs und der Nichtdiskriminierung fußte und im weiteren Verlauf um Mechanismen des Grundrechtsschutzes und der Demokratisierung sowie der Legitimierung der Machtbefugnisse angereichert wurde, sodass sie den Weg einer Konstitutionalisierung beschritten hat.

\section{Im Europarecht}

Die Folgen des Zweiten Weltkrieges stehen in besonderem Kontrast zu denen des Ersten. Auch aufgrund des Kontextes des Kalten Krieges war die Stimmungslage weder dem Gedanken einer Weltgesellschaft noch der Idee einer europäischen Verfassung förderlich. Der Staat trat wieder in den Vordergrund, sowohl als Mitwirkender auf der Weltbühne als auch als Gestalter der jeweiligen nationalen Gesellschaften. Eine Gemeinsamkeit ist jedoch hervorzuheben: die Sehnsucht nach Frieden. Der Wille, einen dauerhafteren Frieden zu etablieren, stand nicht nur am Ursprung der Einrichtung und des Aufbaus der Vereinten Nationen, sondern bildete auch den Motor für die wirtschaftliche europäische Integration und die Europäische Menschenrechtskonvention. Tatsache bleibt bei alldem freilich, dass das Europarecht im Schatten und unter der Aufsicht der Staaten seinen Aufschwung genommen hat. Die anfängliche Zurückhaltung der Rechtsprechung des Europäischen Gerichtshofs für Menschenrechte zeugt deutlich davon.

Doch der beeindruckende Siegeszug namentlich des Verfassungsrechts und insbesondere derjenige der Grundrechte sowie die Verstärkung dieser Entwicklung durch die Demokratisierung mehrerer südeuropäischer Länder in den 1970er Jahren 
führten zuletzt dazu, dass die bestehende Trennung zwischen innerstaatlichem und europäischem Rechtssystem als untragbar angesehen wurde. Der Dialog über den Grundrechtsschutz zwischen dem deutschen Bundesverfassungsgericht sowie dem italienischen Verfassungsgerichtshof auf der einen und dem EuGH auf der anderen Seite ist diesbezüglich bezeichnend. Europa wurde reif für die Idee oder das Projekt einer Konstitutionalisierung.

Die anfängliche Idee geht zweifellos auf das deutsche Bundesverfassungsgericht zurück, genauer gesagt auf dessen Solange I-Rechtsprechung. Sie illustriert, dass die Grundrechte für deutsche Rechtsprechung und Rechtslehre $\mathrm{zu}$ einer Art "Zwangsvorstellung" (»obsession«) ${ }^{42}$ geworden sind: es soll klargestellt werden, dass die europäische Integration nicht voranschreiten könne, wenn bestimmte verfassungsrechtliche Vorgaben unbeachtet bleiben; zu diesen zählt ganz besonders der Schutz der Grundrechte. Der gleiche Gedanke liegt der späteren Solange IIEntscheidung, der Novellierung von Artikel 23 des Grundgesetzes sowie dem Vorschlag der deutschen EU-Ratspräsidentschaft beim EU-Gipfel 1999 in Köln zugrunde, eine Charta der Grundrechte der Europäischen Union zu verfassen.

Doch bleibt der Horizont für die Diskussion dieser Gedanken eine gewisse Zeit lang zunächst auf Deutschland beschränkt, bevor er die europäische Ebene und zuletzt Frankreich erreicht. Im Laufe der 1980er Jahre beschließen die beiden bedeutenden europäischen Gerichte, der EuGH und der EGMR, ihre bisherige Rechtsprechung unter den umfassenden Begriff der Verfassung zu stellen. In seinem Urteil Parti écologiste "Les Verts" gegen Europäisches Parlament ${ }^{43}$ vom 23. April 1986 bezeichnet der EuGH den EGVertrag zum ersten Mal als "Verfassungsurkunde der Gemeinschaft«. Der EGMR seinerseits beruft sich in seinem Loizidou-Urteil ${ }^{44}$ auf die EMRK als Verfassungsinstrument eines europäischen ordre public. Diese Rechtsprechung erfährt breite Resonanz. Insbesondere die angelsächsische ${ }^{45}$ und die französische Rechtslehre greifen den Begriff auf, und zwar so, dass darüber fast dessen Ursprung in Vergessenheit gerät. Manche gehen so weit, in dieser Rechtsprechung eine "Verwandlung des Vertrags in eine Verfassung ${ }^{46}$ oder eine "Richterverfassung « zu sehen, die sich aus der "Weigerung der Staaten, eine wahrhaftige Verfassung auszuarbeiten $\aleph^{47}$, erkläre. Dieser Auffassung nach entspricht die Einführung dieses neuen Signifikanten einer Änderung, einer Verwandlung oder, mit den Worten Joseph Weilers, einer »friedlichen Revolution $«{ }^{48}$ die das Signifikat berühre.

\section{Der Wandel des Bezeichneten: Ist dies eine Verfassung?}

Die These von der Verwandlung ist hochgradig umstritten, sowohl in Bezug auf das Völkerrecht als auch auf die Europäische Union. Daher muss für jeden der beiden Fälle nach der Funktion gefragt werden, die die verwendete staatsrechtliche Terminologie erfüllen soll.

\section{A. Die Begriffswahl im Völkerrecht}

1. Es lässt sich eine gewisse Banalisierung in der Verwendung der Begriffe Verfassung und Konstitutionalisierung beobachten, ohne dass gleichzeitig eine wirkliche Begeisterung für diese Begriffe erkennbar wäre. Diese Verwendung reicht bezüglich 
ihrer Zielsetzung und ihrer Ursprünge nicht über ein gewisses Maß hinaus. Sie bleibt hauptsächlich ein Phänomen der westlichen Rechtslehre, obwohl der Konstitutionalismus weit über sein ursprüngliches Entstehungsgebiet hinaus Ausstrahlungswirkung entfaltet hat und die grundsätzlichen konstitutionellen Ideen und Werte mehr oder weniger weltweit anerkannt werden. ${ }^{49}$ Innerhalb der westlichen Rechtslehre ist es wiederum im Wesentlichen die deutschsprachige, die am aktivsten eine auf verfassungsrechtliche Aspekte gerichtete Behandlung der Charta der Vereinten Nationen und darüber hinaus des Völkerrechts im Allgemeinen vorantreibt. Der zweifelsohne mit der symbolischen Bedeutung, die das Wort Verfassung im nationalstaatlichen Rechtsgedächtnis besitzt, verbundene verfassungsrechtliche Ansatz wird darüber hinaus durch eine Denkweise begünstigt, die den Fokus stärker auf die materiell-rechtliche Dimension eines "Grundgesetzes" richtet, wohingegen die französische Denkweise, um nur sie als Gegenpol zu nennen, einen formal-rechtlichen Betrachtungsansatz vorzieht..$^{50}$ Jedenfalls toben auch weiterhin lebhafte Auseinandersetzungen, selbst unter denjenigen, die am offensten für eine Übertragung der Begrifflichkeiten auf die völkerrechtliche Ebene sind.

Der Übergang vom innerstaatlichen Recht hin zum Völkerrecht ist gleichzeitig der Wechsel von einer Überdeterminierung des Begriffes, durch einen Fächer formeller, institutioneller und materiell-rechtlicher Aspekte, hin zu einer Unterdeterminierung, selbst wenn zweifellos der Bezug $\mathrm{zu}$ der materiell-rechtlichen Dimension des Verfassungsbegriffs überwiegt. Die Unsicherheiten im Hinblick auf die Folgen einer Verwendung dieses Begriffes führen zu einer Zurückhaltung in Bezug auf eben diese Verwendung. Die detailliertesten und am stärksten begründeten Abhandlungen beziehen sich auf die Charta der Vereinten Nationen, was leicht zu erklären ist. Weil die Charta eine politische Organisation begründet, weil das System zur Sicherung der kollektiven Sicherheit wie eine Skizze oder Vorausdeutung eines Gewaltmonopols zugunsten der Vereinten Nationen wirken kann, weil die Charta (quasi-) universelle Geltung entfaltet, kann sie als das geeignetste Mittel zur Verkörperung eines weltweiten Gesellschaftsvertrages erscheinen. Wird die Charta mit einer Verfassung der Staatengemeinschaft gleichgesetzt, behält eine derartige Untersuchung eine institutionell-rechtliche Dimension, die sie jedoch einbüßt, wenn es darum geht, den Faktor zu betrachten, dass sich diese Verfassung nicht auf nur institutionelle Aspekte beschränkt, oder wenn der Begriff in anderen Gebieten verwendet werden soll. Die Herangehensweise betrachtet dann hauptsächlich materiell-rechtliche Aspekte, und gleichzeitig wird der Referenzbegriff unbestimmter.

Die Vorgehensweisen sind nur selten eindeutig und systematisch. Sie sind darüber hinaus auch nicht einheitlich und stützen sich nur in geringem Maße auf klar benannte theoretische Prämissen. In so manchem Fall erfolgt der Rückgriff auf den Verfassungsoder den Konstitutionalisierungsgedanken lediglich aus Bequemlichkeit ${ }^{51}$ oder ist Ausdruck einer Anspielung auf die symbolische Dimension des Verfassungsbegriffs und des Verweises auf einige der grundlegenden, aber ungenau definierten Vorstellungen wie die des Schutzes bestimmter Werte, allen voran der Menschenrechte und der Wahrung des Friedens, oder von mit diesem Schutz mehr oder weniger eng in Zusammenhang stehenden Ideen wie die der Notwendigkeit demokratischer Entscheidungsprozesse, der Hierarchie der Normen, usw. Schließlich scheint sich die Verwendung dieses Begriffs auch im Wettbewerb mit anderen Termini wie dem der Weltgemeinschaft $\mathrm{zu}$ befinden. Dabei entsteht der Eindruck, dass diese 
Begriffsverwendung eine Antwort auf das polyzentrische Wesen des Völkerrechts darstellt und auf die Frage der Einheit des Völkerrechts abzielt. Ein solcher Ansatz ist nicht neu, jedoch verleihen ihm die zunehmenden wechselseitigen Abhängigkeiten (man könnte auch von Globalisierung sprechen, wenn man diesen Begriff nicht auf seine Bedeutung im wirtschaftlichen Sinne beschränkt) eine verstärkte Aktualität. Bei den Überlegungen über eine Strukturierung des Völkerrechts, die mit dieser Erscheinung einhergehen könnte, erlaubt der Gebrauch des Wortes »Verfassung« auf vielsagende Weise, sowohl den Gedanken der Einheit des Völkerrechts aufzuwerfen als auch denjenigen, dass diese Einheit nicht ohne Normenhierarchie zu erreichen ist. Ein solcher Diskussionsansatz ist freilich bei weitem nicht der einzig mögliche, ${ }^{52}$ und selbst wenn dieser Ansatz gewählt wird, führt die Argumentation nicht zwangsläufig über den Verfassungsgedanken, was bedeutet, dass dieser Gedanke entweder durch andere austauschbar ist oder ein Zweifel darüber besteht, ob er für das Völkerrecht geeignet und adaptierbar ist.

32 2. Denn so unbestimmt der Verfassungsbegriff auch sein mag, so ist doch deutlich, dass seine Verwendung für eine Analyse des Völkerrechts - sofern man nicht die Errichtung eines Weltstaates befürwortet - voraussetzt, dass er nicht untrennbar mit dem Begriff des Staates verbunden ist und somit in stichhaltiger Weise auf andere Arten von Gemeinschaften übertragbar ist. ${ }^{53}$ Sie muss des Weiteren davon ausgehen, dass zwischen Völkerrecht und Verfassungsrecht keine unauflösbare Gegensätzlichkeit gesehen wird. Schließlich setzt sie voraus, dass der Gedanke vorstellbar ist, es könne mehrere Verfassungen gleichzeitig geben, ein Gedanke, der offensichtlich Rechtswissenschaftlern aus bundesstaatlich organisierten Staatswesen leichter fällt und zudem Teil von Debatten im spezifischen europäischen Kontext ist, in dessen Rahmen bereits der Gedanke einer »dualen Verfassung ${ }^{54}$ aufgeworfen worden ist. Hier könnte im Übrigen durchscheinen, dass die Debatte um eine Konstitutionalisierung des Europarechts auch leicht schizophrene Züge tragen kann, wenn bedacht wird, dass der verfassungsrechtliche Ansatz auch verwendet wird, um das Gemeinschaftsrecht gegenüber dem Völkerrecht abzugrenzen und Eigenschaften zu benennen, über die das Völkerrecht (noch?) nicht verfügt ${ }^{55}$ oder die es nicht in ihrer Gesamtheit erwerben könnte.

Wenn Martti Koskenniemi die Reflexionen Lauterpachts über die Satzung des Völkerbundes mit dem Begriff Verfassung umreißt, erklärt er dies bezeichnenderweise mit dem politischen Engagement des von ihm Zitierten. ${ }^{56}$ Allgemein kann festgestellt werden, dass die Verwendung des Verfassungsbegriffs in Bezug auf das Völkerrecht stärker auf Rechtssetzung abzielt als beschreibenden Charakter hat. Eine deskriptive Analyse würde rasch an der positivistischen Gegenprobe scheitern, mittels deren mit Leichtigkeit nachgewiesen kann, dass das Völkerrecht - wenn man unterstellt, dass es sich dabei um eine Rechtsordnung handele - nicht die Eigenschaften aufweist, die Vorstellung einer höchsten Norm zuzulassen, und sei diese auch Trägerin von Grundwerten..$^{57}$ Eine präskriptiv ausgerichtete Analyse propagiert die Idee einer Vereinheitlichung des Völkerrechts als Recht einer durch gemeinsame Interessen und universelle Werte miteinander verbundenen Weltgemeinschaft.

Somit handelt es sich um ein politisches Vorhaben, obgleich dies wegen des Gedankens, dass das Völkerrecht ein Flickenteppich ist, den es zu stopfen gilt, leicht aus dem Blick geraten könnte. Indessen verführt die Tatsache, dass diese Vereinheitlichung auf verschiedene Arten erreichbar erscheint, dazu, diesen Prozess zu lenken ${ }^{58}$ oder, falls er 
unter fragwürdigen Vorzeichen geführt wird - beispielsweise als Vereinheitlichung unter dem hegemonialen Einfluss eines einzigen dominierenden Staates -, sich ihm zu widersetzen..$^{59}$ Die Analyse des Völkerrechts unter verfassungsrechtlichen Prämissen ist nur ein Modell unter mehreren. Es gehört zu denjenigen, die zum einen das Völkerrecht in der Genealogie des Kant'schen Gedanken eines kosmopolitischen Rechts sehen, zum anderen aber auch grundlegenden Wert darauf legen, dass das System auf Rechtsnormen basiert (rule-orientated). Der besondere Wert eines solchen Modells besteht darin, dass es versucht, das Vorhaben, auf das es abzielt, zu verbinden mit einer Berücksichtigung des gegenwärtigen Standes der Entwicklung des Rechts, und zwar indem es den Gedanken aufgreift und ihn fördern und schützen will, dass es bestimmte übergeordnete universelle Werte sowie Ansätze einer organisierten Weltgesellschaft und eines Vorrangs des Völkerrechts gibt. Es dient dazu, etwas zu beschreiben, von dem man zugeben muss, dass es noch keinen besonderen Rechtsstatus besitzt - konkret einen obersten Rang, der es unangreifbar machen würde -, bzw. von dem man sich wünschte, es besäße einen derartigen Rechtsstatus. Dieses Modell beeinflusst darüber hinaus die Wahrnehmung des Staats- und des Souveränitätsbegriffs, vielleicht nicht durch das Völkerrecht selbst, aber doch durch die Rechtslehre, welche die auf die Menschenrechte zentrierte inhaltliche Auffüllung und Rangordnung des Völkerrechts mit der Zuschreibung einer materiell-rechtlichen Komponente (insbesondere in Form der Vorgabe einer demokratischen Verfasstheit eines Staatswesens) an den Souveränitätsbegriff verknüpft, was die klassische Sichtweise auf diesen Begriff nicht zwangsläufig vornimmt.

Es bleibt indessen die Aufgabe, zum einen das Wesen der Weltgemeinschaft, um die es gehen soll, ${ }^{60}$ und zum anderen die Denkmuster zu beleuchten, die für die Vergemeinschaftung des Völkerrechts Verwendung finden ${ }^{61}$ - ein Aspekt, bezüglich dessen der verfassungsrechtliche Ansatz noch recht wortkarg bleibt.

\section{B. Die Begriffswahl im Europarecht}

Im Europarecht wurde und wird die Debatte rund um das Thema Konstitutionalisierung äußerst erbittert geführt. Nicht nur eignet sich dieses Gebiet dazu besser als das Völkerrecht, sondern hat auch das Recht selbst den Diskurs über das Recht bereits eingeholt. Grob vereinfacht, können in diesem hochkomplexen Bereich drei Gesichtspunkte erkannt werden: zum einen die Debatte darüber, wie legitim die Übertragung des Verfassungsbegriffs auf die europäische Ebene ist, zum anderen die Neuerungen im positiven Recht und schließlich der Versuch einer wissenschaftlichen Nutzbarmachung des Verfassungsgedankens. Diese drei Gesichtspunkte sind stark miteinander verflochten, wobei sie mehr oder weniger chronologisch aufeinander folgen.

37 1. Unverkennbar ist die Begeisterung für den Diskurs über die Konstitutionalisierung größer als diejenige für die europäische Verfassung selbst. Diese wird oftmals als »bruchstückhaft und unvollständig ${ }^{62}$ angesehen, insbesondere aufgrund des Fehlens einer echten verfassungsgebenden Gewalt und einer eigenen Souveränität. ${ }^{63}$ Die Debatten kreisen zunächst um die mögliche begriffliche Entkoppelung ${ }^{64}$ von Staat und Verfassung - wobei auch bewusst die Unterscheidung zwischen Verfassung im formellen und Verfassung im materiellen Sinne eingesetzt wird - oder um die Frage, ob im Gegenteil diese Begriffe notwendigerweise miteinander verbunden seien, ${ }^{65}$ sodass 
der Dreh- und Angelpunkt zahlreicher wissenschaftlicher Auseinandersetzungen mit diesem Thema letztlich die Frage der staatlichen Souveränität bleibt.

Die Frage, wie aussagekräftig der Rückgriff auf den Verfassungsgedanken für die Beschreibung des europäischen Entwicklungsprozesses ist, hat noch keine abschließende Antwort gefunden. Zwar hat sie die Umrisse eines europäischen »Verfassungsbesitzstandes« klarer zum Vorschein gebracht, allerdings noch nicht alle Ungewissheiten oder Mehrdeutigkeiten ausgeräumt. So werden einerseits die Unterscheidung zwischen der Konstitutionalisierung des Rechts - verstanden als das nationale Recht - und der Konstitutionalisierung des Europarechts ${ }^{66}$ und andererseits die Bedeutung dieser Unterscheidung nicht immer klar herausgestellt. Jean-Claude Gautron hingegen differenziert zwischen "zum einen der Entwicklung der Gemeinschaftsrechtsordnung hin $\mathrm{zu}$ einem Bestand von Verfassungsnormen im materiellen Sinne « und »zum anderen den Anpassungen der nationalen Verfassungen an die Gemeinschaftsrechtsordnung", die »eine Konstitutionalisierung im formellen Sinne darstellen ${ }^{67}{ }^{67}$ Es ist klar, dass die Standpunkte innerhalb des Konstitutionalisierungsdiskurses die Begeisterung, die Faszination, aber auch die Zurückhaltung, bis hin zu offener Feindseligkeit, in Bezug auf die gegenwärtigen Entwicklungen ausdrücken, ${ }^{68}$ aber es liegt auch genauso auf der Hand, dass die Wortwahl nicht neutral ist, sondern - zumindest symbolisch ${ }^{69}$ - Auswirkungen auf das Bezeichnete entfaltet: die Konstitutionalisierung des Europarechts ist demnach mehr als eine bloße Modeerscheinung. Dies auch, weil die Idee, die Europäische Gemeinschaft in einen Bundesstaat umzuwandeln, spürbar nachlässt. ${ }^{70}$ An ihre Stelle tritt ein Gebilde sui generis, nicht wirklich Staat, aber auch nicht wirklich internationale Organisation. ${ }^{71}$ Gerade dieser Gedanke spiegelt sich in der Fortentwicklung des positiven Rechts wider.

2. Durch den Vertrag von Maastricht wurden die Europäische Union und die Unionsbürgerschaft geschaffen, der Vertrag von Amsterdam bezieht sich auf die gemeinsamen Ziele und Werte der Union und der Mitgliedstaaten, und im Rahmen des Vertrags von Nizza haben die EU-Institutionen die Charta der Grundrechte verkündet. 2002/2003 trat ein Konvent zusammen, um für die Union eine Verfassung auszuarbeiten, oder besser gesagt einen Verfassungsvertrag.

Der vom Konvent erstellte Entwurf ist in der Tat vom Geist des Konstitutionalismus durchdrungen, wie er in der Präambel der Erklärung der Menschen- und Bürgerrechte von 1789 zum Ausdruck kommt:

»damit diese Erklärung allen Mitgliedern der Gesellschaft beständig vor Augen ist und sie unablässig an ihre Rechte und Pflichten erinnert; damit die Handlungen der gesetzgebenden wie der ausübenden Gewalt in jedem Augenblick mit dem Endzweck jeder politischen Einrichtung verglichen werden können und dadurch mehr geachtet werden; damit die Ansprüche der Bürger, fortan auf einfache und unbestreitbare Grundsätze begründet, sich immer auf die Erhaltung der Verfassung und das Allgemeinwohl richten mögen . $^{72}$

Hier entsteht der Eindruck, dass durch die Arbeiten des Konvents zum einen eine Verfassung im materiellen Sinne, in deren zweitem Teil die Grundrechte der Charta verankert wurden, und zum anderen eine Verfassung im formellen sinne einer obersten und schriftlich niedergelegten Norm entstanden ist.

41 3. Ist dieser Schluss gerechtfertigt und welche weiteren Schlüsse ergeben sich daraus? Bei dem dritten behandelten Gesichtspunkt steht nun nicht mehr so sehr das politische Projekt im Vordergrund, sondern mehr das Bestreben, in der europäischen Verfassung ein Werkzeug für die wissenschaftliche Betrachtung, eine Analysemethode oder ein 
Bewertungsraster zu sehen. Jean-Claude Gautron greift darauf zurück, wenn er vom "Verfassungsbesitzstand der Union" und ebenso von den "verfassungsrechtlichen Inhalten der im Werden befindlichen Europäischen Union « spricht. ${ }^{73}$ Zum Besitzstand zählt er die Gründungsverträge, wobei er besonders die sich aus der unklaren Gewaltenteilung ergebende Unvollkommenheit des Unionssystems, die beiden Legitimitätsgrundlagen des Parlaments und des Europäischen Rates sowie die Rechtsprechung des EuGH und deren strukturgebende Funktion zugunsten der Gemeinschaftsrechtsordnung hervorhebt. Die Inhalte der im Werden befindlichen Union, die einer Verfassungsebene zugeordnet werden können, werden von ihm bei den Grundrechten und der Unionsbürgerschaft, der Zuständigkeitsverteilung und dem Subsidiaritätsprinzip sowie bei der Normenhierarchie und dem Institutionengefüge verortet.

Der Rückgriff auf die europäische Verfassung führt unweigerlich zu einer Relativierung innerhalb des Diskurses; sie ist mehr ein Messinstrument denn ein Bewertungskriterium. In einem wichtigen Beitrag mit dem Titel »Beobachtungen zur Wissenschaft vom Europarecht. Strukturen, Debatten und Entwicklungsperspektiven der Grundlagenforschung zum Recht der Europäischen Union« nimmt Armin von Bogdandy ${ }^{74}$ eine Bestandsaufnahme der Rechtslehre vor, die zeigt, wie sehr das europäische Recht durch diese beständige Messung am Maßstab verfassungsrechtlicher Begrifflichkeiten Bereicherung und Vertiefung erfahren hat. Diese Feststellung bezieht sich sowohl auf das materielle wie auf das formelle Recht. In letztgenannter Hinsicht wird die Rechtslehre vor allem die Frage nach dem Verfahren für die Ratifizierung beziehungsweise die Zustimmung zu der europäischen Verfassung zu behandeln haben: Soll dieses Verfahren nach den gleichen Vorgaben gestaltet werden - und wenn ja, bis zu welchem Grad - wie denjenigen, die sich aus innerstaatlichem Verfassungsrecht ergeben, oder soll es vor allem den Regeln für eine Änderung des Gründungsvertrages folgen? Soll die Ratifizierung in allen Mitgliedstaaten auf identische Weise erfolgen und, vor allem, soll in ihrem Rahmen, vorher oder gleichzeitig, ein Volksentscheid auf europäischer oder der jeweiligen nationalen Ebene durchgeführt werden?

Dementsprechend bleibt noch $\mathrm{zu}$ fragen, welche Bedeutung dem Phänomen der Konstitutionalisierung beizumessen ist oder, anders ausgedrückt: wie könnte die Stellung der Mitgliedstaaten aussehen, über wieviel Eigenständigkeit würden sie verfügen? Geht es also mithin darum, eine stärker »europäische«, stärker vereinheitlichende Konstitutionalisierung vorzunehmen oder eher eine stärker "nationale«, vielfältigere? Wahrscheinlich wird die Antwort darauf erst am Ende eines langen Entwicklungsprozesses stehen, denn "Konstitutionalisierung beschreibt eine Richtung, einen Verlauf, und sicher nicht einen abgeschlossenen Zustand «, ${ }^{75}$ oder, um nochmal Magritte zu zitieren: dies ist und ist keine Verfassung.

\section{BIBLIOGRAPHIE}

Alland, D. (2002): »De l'ordre juridique international«, Droits, 1, Nr. 35, S. 79-101. 
Alston, Ph. (2002): »Resisting the Merger and Acquisition of Human Rights by Trade Law: A Reply to Petersmann«, EJJL, 4, S. 815-844.

Bauer, H. (2000): »Europäisierung des Verfassungsrechts«, JBI, S. 750-763.

Beaud, O. (1998): „Fédéralisme et souveraineté. Notes pour une théorie de la Fédération«, $R D P, 1$, S. $83-122$.

Bernhardt, R. (2002): „Comment on Article 103«, in: Simma, B. et al. (Hg.): The Charter of the United Nations: A Commentary, 2. Aufl.

Böckenförde, E.-W. (1999): »Welchen Weg geht Europa?«, in: ders.: Staat, Nation, Europa, Frankfurt am Main: Suhrkamp, S. 68-102.

Bogdandy, A. von (2001): »Beobachtungen zur Wissenschaft vom Europarecht: Strukturen, Debatten und Entwicklungsperspektiven der Grundlagenforschung zum Recht der Europäischen Union«, Der Staat, 40 (1), S. 3-43.

Bogdandy, A. von (2002): Europäisches Verfassungsrecht, Berlin: Springer.

Cass, D. Z. (2001): »The `Constitutionalization« of lnternational Trade Law: Judicial NormGeneration as the Engine of Constitutional Development in International Law«, EJIL, S. 39-75.

Combacau, J. (1986): »Le droit international: bric-à-brac ou système?«, Arch Phil. Dr., S. 85-105.

Constantinesco, V. (2000): "La constitutionnalisation de l'Union européenne«, in: Rideau, J. (Hg.): De la communauté de droit à l'union de droit. Continuités et avatars européens, Paris: LGDJ, S. 133-152.

Daillier, V. P. / Pellet, A. (2002): Droit international public, Paris: LGDJ.

Dehousse, R. (2002): Une Constitution pour l'Europe?, Paris: Presses de Sciences Po.

Delmas-Marty, M. (1998): Trois défis pour un droit mondial, Paris: Seuil.

Duguit, L. (2003): L'État, le droit objectif et la loi positive, Paris: Dalloz.

Dupuy, P.-M. (2002): »L'unité de l'ordre juridique international«, RCADI, 297, S. 15-489.

Epiney, A. (2001): »Européanisation et mondialisation du droit: convergences et divergences«, in: Morand, C.-A. (Hg.): Le droit saisi par la mondialisation, Brüssel: Bruylant, S. 145-170.

Falk, R. A. et al. (Hg.) (1993): The Constitutional Foundations of World Peace, New York: State University of New York Press.

Fassbender, B. (1998): »The United Nations Charter as Constitution of the International Community«, Columbia Journal of Transnational Law, 36, Nr. 3, S. 531-617.

Favoreu, L. (1990): »Le droit constitutionnel, droit de la Constitution et constitution du droit«, RFDC, S. 71-89.

Frowein, J. (1994): »Reactions by Not Directly Affected States to Breaches of Public International Law«, RCADI, 248, S. 345-438.

Gautron, J.-C. (2000): „La qualification de l'ordre juridique communautaire«, in: Gaudin, H. (Hg.): Droit constitutionnel, droit communautaire. Vers un respect constitutionnel réciproque?, Paris / AixMarseille: Economica / PUAM.

Gautron, J.-C (2003): »Rapport de synthèse: La `constitution européenne`: une Constitution?, Journée d'études du 23 mars 2003«, RAE-LEA, 2001-2002 (6), S. 744-754.

Gerkrath, J. (1997): L'émergence d'un droit constitutionnel pour l'Europe, Brüssel: Éd. de l'Université de Bruxelles. 
Grewe, C. (1999): »Demokratie ohne Volk oder Demokratie der Völker? Zur Demokratiefähigkeit der Europäischen Union«, in: Drexl, J. / Kreuzer, K. F. / Scheuing, D. H. / Sieber, U. (Hg.): Europäische Demokratie, Baden-Baden: Nomos, S. 59-70.

Grewe, C. / Ruiz Fabri, H. (1995): Droits constitutionnels européens, Paris: PUF.

Grimm, D. (1999): »Ohne Volk keine Verfassung«, Die Zeit, 18. März.

Howse, R. / Nicolaïdis, K. (2001): »Legitimacy and Global Governance: Why Constitutionalizing the WTO is a Step too Far «, in: Porter, R. B. (Hg.): Efficiency, Equity, and Legitimacy: The Multilateral Trading System at the Millenium, Washington DC: The Brookings Institution, S. 227-252.

Hurrell, A. (2000): »International Law and the Changing Constitution of International Society«, in: Byers, M. (Hg.): The Role of Law in International Politics: Essays in International Relations and International Law, Oxford: Oxford UP, S. 327-350.

Jacqué, J.-P. (1991): »Le rôle du droit dans l'intégration européenne«, Philosophie politique, S. 119133.

Jouanjan, V. O. (2003): „Ce que >donner une Constitution à l'Europe` veut dire«, Cités, 1, Nr. 13, S. 21-35.

Jouannet, E. (2000): »Regards sur un siècle de doctrine française du droit international«, AFDI, S. $1-57$.

Jouannet, E. (2003): „L'idée de communauté humaine à la croisée de la communauté des Etats et de la communauté mondiale«, Arch. Phil. Dr., 47, S. 191-232.

Kirchhof, P. (1992): »Der deutsche Staat im Prozess der europäischen Integration«, in: Isensee, J. / Kirchhof, P. (1992): Handbuch des Staatsrechts, Bd. 7, Heidelberg: Müller Verlag.

Kolb, R. (2003): Réflexions de philosophie du droit international, Brüssel: Bruylant.

Koskenniemi, M. (2001): The Gentle Civilizer of Nations, Cambridge: Cambridge UP.

Koskenniemi, M. (2003): »Comment «, in: Byers, M. / Nolte, G. (Hg.): United States Hegemony and the Foundations of International Law, Cambridge: Cambridge UP, S. $91 \mathrm{ff}$.

Krajewski, M. (2001): „Democratic Legitimacy and Constitutional Perspectives of WTO Law«, JWT, S. 167-186.

Krisch, N. (2003): »Hierarchy, equality and US Predominance«, in: Byers, M. / Nolte, G. (Hg.) (2003): United States Hegemony and the Foudations of International Law, Cambridge: Cambridge University Press, S. 135-175.

La »constitution européenne«: une Constitution?, Actes de la Journée d'études constitutionnelles du 28 mars 2003, RAE-LEA, 2001-2002 (6).

Lauterpacht, H. (1936a): »The Covenant as the Higher Law«, BYIL, S. 54-65.

Lauterpacht, H. (1936b): » Neutrality and Collective Security«, Politica, 2, S. 133-155.

Mayer, F. C. (2003): »La Charte européenne des droits fondamentaux et la Constitution européenne«, Revue trimestrielle de droit européen, 39, H. 2, S. 175-196.

Mirkine-Guetzevich, B. (1933): Droit constitutionnel international, Paris: Sirey.

Mockle, D. (Hg.) (2002): Mondialisation et État de droit, Brüssel: Bruylant.

Nabli, B. (2003): „Européanisation et constitutionnalisation du droit en France: un essai de synthèse à la lumière de la doctrine«, in: Ziller, J. (Hg.): L'européanisation des droits constitutionnels à la lumière de la Constitution pour l'Europe, Paris: L'Harmattan, S. 69-84. 
Pernice, I. / Mayer, F. C. (2000): »De la constitution composée de l'Europe«, Revue trimestrielle de droit européen, 36 (4), S. 623-648.

Petersmann, E. U. (2002a): „Constitutionalism and WTO Law: From a State-Centered Approach Towards a Human Rights Approach in International Economie Law«, in: Kennedy, D. L. M. / Southwick, J. O. (Hg.): The Political Economy of International Law, Cambridge: Cambridge UP, S. 3267.

Petersmann, E. U. (2002b): »Time for a United Nations `Global Compact « for Integrating Human Rights into the Law of Worldwide Organizations: Lessons from European integration «, EJIL, 13, Nr. 3, S. 621-659.

Rasmussen, H. (1991): »The Court of Justice of the European Communities and the process of Integration«, in: Orban, E. /Favoreu, L. (Hg.): Fédéralisme et Cours suprêmes / Federalism and Supreme Courts, Brüssel: Bruylant.

Rideau, J. (1990): „Constitution et droit international dans les États membres des Communautés européennes«, RFDC, 2, S. 259-296.

Romano, S. (1975 [1918]): L'ordre juridique, Paris: LGDJ.

Ruiz Fabri, H. (1992): „Genèse et disparition de l'Etat à l'époque contemporaine«, AFDI, S. 153-178.

Ruiz Fabri, H. (2001): »L'ordre public en droit international«, in: Redor, M.-J. (Hg.): L'ordre public: ordre public ou ordres publics? Ordre public et droits fondamentaux, Brüssel: Bruylant, S. 85-108.

Scelle, G. (1932): Précis de droit des gens, Paris: Sirey.

Schloemann, H. L. / Ohlhoff, S. (1998): »Rational Allocation of Disputes and 'Constitutionalisation`: Forum Choice as an Issue of Competence«, in: Cameron, J. / Campbell, K. (Hg.) (1998): Dispute Resolution in the World Trade Organisation, London: Cameron May, S. 302-329.

Schloemann, H. L. / Ohlhoff, S. (1999): ")Constitutionalization<, and Dispute Settlement in the WTO: National Security as an Issue of Competence«, AJIL, 93, H. 2, S. 424-451.

S.F.D.I. (2003): La juridictionnalisation du droit international, Paris: Pedone.

Sicilianos, A. (2000): L'ONU et la démocratisation de l'État, Paris: Pedone.

Simma, B. (1994): »From bilateralism to community interest in international law«, RCADI, 250, S. 217-384.

Simon, D. (2001): Le système juridique communautaire, 3. Aufl., Paris: PUF.

Skubiszewski, K. (1983): »Remarks on the Interpretation of the United Nations Charter «, in: Bernard, R. (Hg.): Völkerrecht als Rechtsordnung - internationale Gerichtsbarkeit - Menschenrechte, Festschrift für Hermann Mosler, Berlin u. a.: Springer.

Tomuschat, Ch. (1993): „Obligations Arising for States without or against Their Will«, RCADI, 241, S. 195-374.

van de Kerchove, M. (2002): De la pyramide au réseau. Pour une théorie dialectique du droit, Brüssel: Publications des FUSL.

Verdross, A. (1923): Die Einheit des rechtlichen Weltbildes auf der Grundlage der Völkerrechtsverfassung, Tübingen: Mohr.

Verdross, A. (1926): Die Verfassung der Völkerrechtsgemeinschaft, Wien: Springer.

Verdross, A. / Simma, B. (1976): Universelles Völkerrecht: Theorie und Praxis, Berlin: Duncker \& Humblot. 
Weiler, J. H. (1995): „Une révolution tranquille. La Cour de Justice des Communautés européennes et ses interlocuteurs«, Politix, 32, S. 119-138.

Ziller, J. (Hg.) (2003): L'européanisation des droits constitutionnels à la lumière de la Constitution pour l'Europe, Paris: L'Harmattan.

\section{NOTES}

1. Gautron (2003), S. 744.

2. Mirkine-Guetzevich (1933).

3. Rideau (1990).

4. Ziller (2003); Bogdandy (2002); Bauer (2000), S. 750 f.

5. Pernice / Mayer (2000), S. 623 f.

6. Gautron (2000), S. 365.

7. Constantinesco (2000), S. 137; Fassbender (1998); Dupuy (2002), insb. S. 215-244.

8. Favoreu (1990), S. $71 \mathrm{f}$.

9. Gautron (2003), S. 744.

10. Bemerkenswert ist an dieser Stelle die gelungene Begriffsschöpfung der "Juridiktionalisierung« durch die Völkerrechtslehre, so z. B. bei der Französischen Vereinigung für Völkerrecht (S.F.D.I.), s. SFDI (2003).

11. S. u. a. Petersmann (2002a), (2002b).

12. Kolb (2003), S. 23.

13. Womit eine Verneinung des Rechtscharakters des Völkerrechts zum Ausdruck gebracht wird, beziehungsweise eine Verneinung des Völkerrechts selbst. In gewisser Hinsicht ist der Verfassungsgedanke diesen Ansätzen nicht fremd, wenngleich er auch nur im Hintergrund bleibt. Die Negierung des Völkerrechts wird aus der Tatsache abgeleitet, dass es keine den Staaten übergeordnete Institutionen gibt, die Zwang auf die Staaten ausüben könnten, mit anderen Worten, dass keine überstaatliche Organisation existiert, auf die der Verfassungsgedanke anwendbar sein könnte, wenn man sich die Mühe machte, die Kriterien zu ermitteln, die eine solche Organisation dafür erfüllen müsste. Weniger absolut betrachtet kann die These vom nicht-rechtlichen Charakter des Völkerrechts auch zum Verfassungsbegriff zurückführen, wenn sie das Völkerrecht eng begreift als das öffentliche Recht der Außenbeziehungen eines Staates. Die Verfassung, um die es dann geht, ist allerdings die nationale Verfassung. S. Daillier / Pellet (2002), S. 85-88.

14. Kolb hebt richtigerweise hervor, dass die allgemeinen Vorlesungen an der Haager Akademie für Völkerrecht in den Zwischenkriegsjahren fast stets den Titel trugen »Allgemeine Bestimmungen des Friedensrechts«, s. Kolb (2003), S. 24.

15. Kolb (2003), S. 24.

16. So z. B. Romano (1975 [1917]).

17. Scelle (1932); Duguit (2003).

18. Verdross (1923); Verdross (1926); s. auch Gerkrath (1997), S. 99-113.

19. Alland (2002).

20. Jouannet (2000).

21. Dupuy (2002), S. 227.

22. Skubiszewski (1983), S. 891.

23. Dupuy (2002), S. 228.

24. Koskenniemi (2001), S. $376 \mathrm{f}$.

25. Lauterpacht (1936a), S. 55, zit. bei Koskenniemi (2001), S. 376. Hier sei angemerkt, dass der von Lauterpacht verwendete Begriff »the Higher Law« der von den Federalist Papers in den 
Vereinigten Staaten verwendete Begriff ist, um die Verfassung zu bezeichnen. S. Fassbender (1998), S. 534. Artikel 20 der Völkerbundsatzung sah vor: »1. Die Bundesmitglieder erkennen, ein jedes für sein Teil, an, daß die gegenwärtige Satzung Verpflichtungen und Einzelverständigungen aufhebt, die mit ihren Bestimmungen unvereinbar sind, und verpflichten sich feierlich, solche in Zukunft nicht mehr einzugehen. 2. Hat ein Mitglied vor seinem Eintritt in den Bund Verpflichtungen übernommen, die mit der Satzung unvereinbar sind, so hat es die Pflicht, unverzüglich Maßnahmen zur Lösung dieser Verpflichtungen zu ergreifen.«

26. Lauterpacht (1936b), S. 133, zit. bei Koskenniemi (2001), S. 377.

27. Fassbender (1998); Tomuschat (1993); Bernhardt (2002), S. 1292; Frowein (1994); Simma (1994).

28. Verdross / Simma (1976).

29. Dupuy (2002), S. 228.

30. Hier findet die alte Unterscheidung zwischen Verträgen als Gesetz und Verträgen als Übereinkommen einen Nachhall, obgleich eine solche Unterscheidung umstritten ist. Das Völkerrecht selbst greift sie nicht auf, es sieht keine unterschiedliche Rechtsstellung für die betreffenden Vertragsarten vor.

31. Falk (1993).

32. Krisch (2003), S. 150 f.

33. Grewe / Ruiz Fabri (1995), S. $21 \mathrm{f}$.

34. Ruiz Fabri (2001).

35. Sicilianos (2000); Mockle (2002).

36. Ruiz Fabri (1992).

37. Koskenniemi (2001), S. 512.

38. Epiney (2001), insb. S. 164.

39. Krajewski (2001).

40. Cass (2001).

41. Hierzu s. z. B. Schloemann / Ohlhoff (1998) und (1999); Petersmann (2002a) und (2002b). Für eine Entgegnung s. Alston (2002). Für eine Gegenposition, die die Risiken einer solchen "Konstitutionalisierung« betont, s. Howse / Nicolaïdis (2001).

42. Der Begriff wird verwendet von Mayer (2003), insb. S. 178.

43. Rs. 294/83, Slg., S. 1365.

44. Loizidou / Türkei, Urt. v. 23. März 1995, Preliminary Objections, Serie A, Nr. 310.

45. S. insb. das bei Bogdandy (2001), S. 3 f., zitierte Literaturverzeichnis.

46. Zum EuGH s. Rasmussen (1992), insb. S. 201.

47. Jacqué (1991), insb. S. 132.

48. Weiler (1995).

49. Fassbender (1998), S. 555.

50. Dupuy (2002), S. 230.

51. Der Verfassungsbegriff scheint manchmal bloß als Lockmittel verwendet zu werden, ohne im Weiteren Gegenstand einer eingehenderen Analyse $\mathrm{zu}$ sein. So tragen beispielsweise die Schlussbemerkungen Andrew Hurrells zum Symposium The Role of Law in International Politics zwar den Titel "International Law and the Changing Constitution of International Society" ([2000], S. 327), doch wird weder der Begriff noch der dahinter stehende Gedanke im inhaltlichen Teil dieses Beitrags wieder aufgegriffen.

52. Ost / van de Kerchove (2002), insb. S. 125-183.

53. Fassbender (1998), S. $561 \mathrm{f}$.

54. Pernice / Mayer (2000), S. 623.

55. Simon (2001), S. $73 \mathrm{f}$.

56. Koskenniemi (2001), S. $376 \mathrm{f}$.

57. Combacau (1986). 
58. Delmas-Marty (1998).

59. Koskenniemi (2003), S. 98.

60. Jouannet (2003).

61. Kolb (2003), S. 23 f.

62. Constantinesco (2000), S. 152.

63. Jouanjan (2003).

64. Zu möglichen Wegen für eine solche Entkoppelung s. Jouanjan (2003), S. 29; Beaud (1998), S. 89; Pernice / Mayer (2000), S. $623 \mathrm{f}$.

65. Dieser Aspekt wird besonders betont unter anderen bei Grimm (1999); Kirchhof (1992), S. 855 f. Dagegen: s. Grewe (1999), S. 59 f.

66. Nabli (2003), S. 69 f.

67. Gautron (2000), S. 365.

68. La »constitution européenne«: une Constitution?, S. 655-753.

69. In diesem Sinne Jouanjan (2003), S. 33, und Mayer (2003), S. 194 f.

70. Für diese Idee s. insb. die Rede Joschka Fischers an der Humboldt-Universität in Berlin am 12. Mai 2000; abgedruckt bei Dehousse (2002), S. 238.

71. Über die strukturellen Probleme dieses Gebildes, das auf einer Abspaltung zwischen dem Wirtschafts- und Währungsbereich und der Weltgesellschaft beruht, s. Böckenförde (1999).

72. Präambel der Erklärung der Menschen- und Bürgerrechte vom 26. August 1789.

73. Gautron (2003), S. 744 f.

74. Bogdandy (2001), S. 3 f.

75. Gautron (2000), S. 366

INDEX

Mots-clés : constitutionnalisation, droit international, droit européen

Schlüsselwörter : Konstitutionalisierung, Völkerrecht, Europarecht

\section{AUTEURS}

\section{CONSTANCE GREWE}

Constance Grewe ist emeritierte Professorin an der Universität Straßburg. Nähere Informationen finden Sie hier.

\section{HÉLÈNE RUIZ FABRI}

Hélène Ruiz Fabri ist Direktorin des Max Planck-Instituts für Vefahrensrecht in Luxemburg. Nähere Informationen finden Sie hier. 